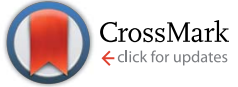

Cite this: RSC Adv., 2017, 7, 3470

Received 8th November 2016 Accepted 30th November 2016

DOI: 10.1039/c6ra26479d

www.rsc.org/advances

\section{Investigations on the effect of carriers on meta- aramid fabric dyeing properties in supercritical carbon dioxide}

\begin{abstract}
Huan-Da Zheng, Juan Zhang, Jun Yan and Lai-Jiu Zheng*
Dyeing of meta-aramid fabric with dimethyl terephthalate, ethyl alcohol and CINDYE DNK as carriers was investigated in supercritical carbon dioxide fluid. The effect of different carriers on the dyeing properties of meta-aramid fabric with Disperse Blue Black 79, Disperse Rubine H-2GL, and Disperse Yellow EC-3G was examined by measuring the color strength with different dyeing temperatures, dyeing pressures, dyeing times, dye concentrations, carrier concentrations and carbon dioxide flows. The results showed that the carriers added in supercritical carbon dioxide were beneficial to the diffusibility of disperse dye molecules into the amorphous region of the meta-aramid fiber, thereby improving its dyeability. In these three carriers, the $K / S$ values of the dyed meta-aramid samples with ethyl alcohol were higher than the samples with dimethyl terephthalate, and the greatest improvement on K/S values appeared upon adding CINDYE DNK in supercritical carbon dioxide. In addition, the dyed meta-aramid fabrics in supercritical carbon dioxide presented good wash fastness, rub fastness and light fastness, which was rated at 4-5.
\end{abstract}

\section{Introduction}

Supercritical carbon dioxide dyeing is an anhydrous dyeing process with numerous advantages in comparison with conventional aqueous dyeing, such as no effluent discharged into the environment, short production process and recycling of dyes and carbon dioxide, as well as energy preservation. ${ }^{1-4}$ Thus, it is an environmentally benign process to use supercritical carbon dioxide dyeing to replace the conventional aqueous dyeing methods as more stringent environmental protection laws have been enacted in most countries. ${ }^{5}$ Particularly, under the implementation of Action Plan for Water Pollution Prevention, the State Council of the People's Republic of China plans that by 2020 , the water quality in $70 \%$ of the drainage areas of the Yangtze, the Yellow, the Pearl, the Songhua, the Huaihe, the Haihe and the Liaohe will be excellent. In order to solve the severe water pollution, there are considerable research activities and experiments on supercritical carbon dioxide dyeing, from laboratory scale to pilot scale.

In supercritical state, the viscosity and diffusivity of carbon dioxide are like that of a gas, whereas the density is close to that of a liquid, resulting in this solvent easily tuned by controlling temperature and pressure. ${ }^{6,7}$ The dissolving property of supercritical carbon dioxide towards disperse dyes, as well as the swelling and plasticisation towards hydrophobic polymers, make this fluid suitable for the dyeing of polyester and other

Liaoning Provincial Key Laboratory of Ecological Textile, Dalian Polytechnic University, Dalian 116034, Liaoning, China. E-mail: fztrxw@dlpu.edu.cn; Fax: +86411-86323438; Tel: +86-411-86323511 synthetic fabrics. ${ }^{8}$ At present, coloration of polyethylene terephthalate, ${ }^{9-11}$ polylactide, ${ }^{12}$ polyamide 6 and 66 (ref. 13-15) with disperse dyes in supercritical carbon dioxide has already acquired satisfactory effect. Moreover, different dyeing explorations on natural fibers in supercritical carbon dioxide have also been documented, such as, disperse dye for pretreated/ modified cellulose fibers, ${ }^{16}$ and disperse reactive dyes for wool, silk and cotton fibers. ${ }^{17,18}$

meta-Aramid fiber belongs to the category of aromatic polyamides: it is a manufactured fiber in which the fiber-forming substance is a long-chain synthetic polyamide in which at least $85 \%$ of the amide linkages are attached directly to two aromatic rings. ${ }^{19}$ This aromatic polyamide fiber, which was firstly synthesized in 1960 and introduced in commercial applications in 1967 by Du Pont under the trademark Nomex®, is characterized by its excellent thermostability, flame retardance, electric insulativity, and radiation resistance. ${ }^{20}$ meta- $^{-}$ Aramid fiber is currently used for heat-resistant filter materials, electrical insulating materials, honeycomb structure materials as well as flame-retardant materials. It also found application in the fields of protective garments, such as spacesuits, firefighter uniforms, racing wears as well as work clothes for oil field, which greatly increases the dyeing demand. Thus, it is necessary to dye meta-aramid fiber in different colors to meet the ever growing needs. However, a high degree of macromolecular orientation and dense crystal structure due to the numerous hydrogen bonds between amide groups in adjacent chains result in the extremely poor dyeability of meta-aramid fiber. ${ }^{21,22}$

Carrier, as a type of accelerant, is mainly used in the dyeing or printing of synthetic fibers with disperse dyes by acting as 
Table 1 Toxicity of the carriers used in meta-aramid dyeing ${ }^{27}$

\begin{tabular}{|c|c|c|c|}
\hline Carriers & Formula & $\begin{array}{l}\text { Molecular } \\
\text { weight }\end{array}$ & Toxicity \\
\hline Benzyl alcohol & $\mathrm{C}_{7} \mathrm{H}_{8} \mathrm{O}$ & 108.13 & $\begin{array}{l}\text { Harmful (oral rat } \\
\mathrm{LD}_{50}: 1230 \mathrm{mg} \mathrm{kg}^{-1} \text { ) }\end{array}$ \\
\hline$N, N$-Diethyl- $m$-toluamide & $\mathrm{C}_{12} \mathrm{H}_{17} \mathrm{NO}$ & 191.27 & $\begin{array}{l}\text { Harmful (oral rat } \\
\mathrm{LD}_{50}: 1950 \mathrm{mg} \mathrm{kg}^{-1} \text { ) }\end{array}$ \\
\hline$N$-Methylformanilide & $\mathrm{C}_{8} \mathrm{H}_{9} \mathrm{NO}$ & 135.16 & $\begin{array}{l}\text { Harmful (oral rat } \\
\mathrm{LD}_{50}: 4000 \mathrm{mg} \mathrm{kg}^{-1} \text { ) }\end{array}$ \\
\hline
\end{tabular}

a plasticising agent to reduce the glass transition temperature $\left(T_{\mathrm{g}}\right)^{23}$ Theoretically, in the dyeing process, carrier can be absorbed by fibers through polar and non-polar forces of interaction, hydrogen bonding and hydrophobic interaction. The flexibility of polymer molecular chains is improved, and the free volume of the fiber is also promoted, leading to the increase of dye exhaustion. Hence, dyeing of polyester, polyphenylene sulphide as well as acrylic fibers with carriers has been conducted..$^{24}$ It is also technically feasible to use carriers in the dyeing of meta-aramid to obtain the deep shades and various hues. ${ }^{25}$ At present, the carrier dyeing of meta-aramid fiber with benzyl alcohol, $N, N$-diethyl- $m$-toluamide, and $N$-methylformanilide in water have been reported extensively for their good carrier dyeing results. ${ }^{26}$ On the other hand, carriers also present certain drawbacks in the industrial-scale production since carriers proposed in the literature are found to have high toxicity and strong irritant odor (listed in Table 1). Simultaneously, the dyeing process also causes numerous environmental concerns because of residual carriers in wastewater. But unfortunately, to date, there are less data for the ecofriendly dyeing approach of meta-aramid fiber using supercritical carbon dioxide as dyeing medium and the suitable nontoxic carriers in the dyeing procedure. Also, no information has been available on the functional mechanism of carriers in supercritical carbon dioxide.
In the present work, dyeing properties of meta-aramid were investigated with Disperse Blue Black 79, Disperse Rubine H-2GL, and Disperse Yellow EC-3G in supercritical carbon dioxide. Nontoxic carriers, dimethyl terephthalate (DMT), ethyl alcohol and CINDYE DNK were adopted for the supercritical carbon dioxide dyeing process. The effects of dyeing temperature, dyeing pressure, dyeing time, dye concentration, carbon dioxide flow as well as carrier concentration on the dyeability of meta-aramid fiber were discussed. Moreover, the functional mechanism of carriers in supercritical carbon dioxide was also proposed.

\section{Experimental}

\subsection{Materials and chemicals}

meta-Aramid fabrics ( $m$-aramid fiber 93\%, $p$-aramid fiber 5\%, organic conductive fiber $2 \%, 2 / 1$ twill weave, weft and warp 32s/2) were supplied by Dandong Unik Textile Co., Ltd. (China). Disperse Blue Black 79, Disperse Rubine H-2GL, and Disperse Yellow EC$3 \mathrm{G}$ without any addition of surfactants and salts was supplied by Zhejiang Longsheng Group Co., Ltd. (China) and used without further purification. Dimethyl terephthalate was obtained from Sinopharm Chemical Reagent Co., Ltd. (China). CINDYE DNK was purchased from Ningbo Bozzetto Group (China), and its formula and ingredients are unknown due to commercial confidentiality. Ethyl alcohol and sodium hydroxide were obtained as analytical reagent grade materials from Tianjin Kemiou Chemical Reagent Co., Ltd. (China). Carbon dioxide gas (99.9 vol\%) obtained from China Haohua (Dalian) Research \& Design Institute of Chemical Industry Co., Ltd. was used for supercritical dyeing. The dyes and carriers used in supercritical carbon dioxide dyeing were listed in Tables 2 and 3, respectively.

\subsection{Apparatus and procedures}

2.2.1 Scouring. Prior to dyeing, meta-aramid fabric was scoured in a solution containing $3 \mathrm{~g} \mathrm{l}^{-1}$ sodium hydroxide and $2 \mathrm{~g}$ $1^{-1}$ soap powder with a mass/liquor ratio of $1: 20$ to remove spinning and knitting waxes and oils from the fibers. The bath

Table 2 The dyes used in supercritical carbon dioxide dyeing

\begin{tabular}{|c|c|c|c|c|}
\hline Dye & Color index & Formula & Molecular weight & Chemical structure \\
\hline Disperse Blue Black 79 & 11344 & $\mathrm{C}_{23} \mathrm{H}_{25} \mathrm{BrN}_{6} \mathrm{O}_{10}$ & 625.38 & \\
\hline Disperse Rubine H-2GL & 11338 & $\mathrm{C}_{23} \mathrm{H}_{26} \mathrm{ClN}_{5} \mathrm{O}_{7}$ & 519.93 & \\
\hline Disperse Yellow EC-3G & 47020 & $\mathrm{C}_{18} \mathrm{H}_{11} \mathrm{NO}_{3}$ & 289.28 & \\
\hline
\end{tabular}


Table 3 The carriers used in supercritical carbon dioxide dyeing

\begin{tabular}{|c|c|c|c|}
\hline Carriers & Formula & $\begin{array}{l}\text { Molecular } \\
\text { weight }\end{array}$ & Toxicity \\
\hline DMT & $\mathrm{C}_{10} \mathrm{H}_{10} \mathrm{O}_{4}$ & 194.19 & $\begin{array}{l}\text { Non-toxicity (oral rabbit } \\
\mathrm{LD}_{50}: 10000 \mathrm{mg} \mathrm{kg}^{-1} \text { ) }\end{array}$ \\
\hline Ethyl alcohol & $\mathrm{C}_{2} \mathrm{H}_{6} \mathrm{O}$ & 46.07 & $\begin{array}{l}\text { Non-toxicity (oral rabbit } \\
\mathrm{LD}_{50}: 7060 \mathrm{mg} \mathrm{kg}^{-1} \text { ) }\end{array}$ \\
\hline CINDYE DNK & $\begin{array}{l}\text { Aromatic amide } \\
\text { compound }\end{array}$ & $\begin{array}{l}\text { Not } \\
\text { available }\end{array}$ & $\begin{array}{l}\text { Non-toxicity (oral rabbit } \\
\mathrm{LD}_{50}:>5000 \mathrm{mg} \mathrm{kg}^{-1} \text { ) }\end{array}$ \\
\hline
\end{tabular}

was raised to $100{ }^{\circ} \mathrm{C}$ for $30 \mathrm{~min}$. meta-Aramid fabric was then rinsed with cold water at $20^{\circ} \mathrm{C}$ and dried at ambient temperature.

2.2.2 Supercritical carbon dioxide dyeing. In the dyeing process, two different injection modes were used as carriers were divided into two categories, liquid and solid. The liquid carriers, ethyl alcohol or CINDYE DNK, were stored in a cosolvent tank. meta-Aramid fabric was wrapped around a porous beam and placed into a dyeing vessel. Disperse dyes with a ratio of $1.5 \%$ to $5.5 \%$ o.m.f. (on the mass of fabric), was packed into a dye cylinder and placed into a dye vessel. After the dyeing vessel and the dye vessel were sealed, carbon dioxide gas in a cylinder filtered with a purifier was firstly introduced into the dyeing system. The gas was then liquefied by employing a refrigerator and was pressurized to above the critical pressure (7.38 MPa) using a high-pressure pump. Simultaneously, the carrier in a co-solvent tank was injected into the dyeing system with a concentration of $1 \%$ to $5 \%$ o.m.f. (on the mass of carbon dioxide) by a co-solvent pump. The carbon dioxide was homogeneously mixed with the liquid carrier in a mixer and heated to above the critical temperature $\left(31.10{ }^{\circ} \mathrm{C}\right)$ with a heat exchanger. When the supercritical state was attained, the solid dyes were dissolved in supercritical carbon dioxide and flowed into the dyeing vessel where meta-aramid fabric could be dyed. The solid carrier, dimethyl terephthalate was packed into a dye cylinder with disperse dye with a ratio of $1 \%$ to $5 \%$ o.m.f. (on the mass of fabric). In the dyeing process, dimethyl terephthalate was dissolved with dyes together in supercritical carbon dioxide, and completed the dyeing for meta-aramid fiber in the dyeing vessel with the circulation of supercritical carbon dioxide fluid.

After the request condition was achieved, the meta-aramid fabric was dyed for $10 \mathrm{~min}$ to $90 \mathrm{~min}$ at dyeing temperatures, pressures and carbon dioxide flows ranging from $80{ }^{\circ} \mathrm{C}$ to $160{ }^{\circ} \mathrm{C}, 18 \mathrm{MPa}$ to $34 \mathrm{MPa}$, and $10 \mathrm{~g} \mathrm{~min}^{-1}$ to $50 \mathrm{~g} \mathrm{~min} \mathrm{~min}^{-1}$, respectively. During the dyeing, the temperatures of the dye vessel and dyeing vessel were keep constant by means of a heat compensating jacket. The dyed fabric was then extracted with fresh carbon dioxide for $20 \mathrm{~min}$ at $70{ }^{\circ} \mathrm{C}$ and $16 \mathrm{MPa}$ to remove the unfixed dyes and the carriers from the fabric and the pipelines. Finally, the carbon dioxide fluid and the dissolved dyes in the dyeing system were separated with a separator, and recycled at pressures and temperatures ranging from $3 \mathrm{MPa}$ to $4 \mathrm{MPa}$ and from $25{ }^{\circ} \mathrm{C}$ to $40{ }^{\circ} \mathrm{C}$, respectively. The dyed metaaramid fabric was removed and used for further analysis when the dyeing process was finished. The apparatus used in this study is shown schematically in Fig. 1.
2.2.3 Colorimetric measurements. The reflectance of the folded two-ply of the dyed sample was measured in the range of $380 \mathrm{~nm}$ to $720 \mathrm{~nm}$ by employing a Color-Eye 7000A spectrophotometer (X-rite, America). The color strength $(K / S$ value) was calculated from the reflectance at the wavelength of maximum absorption for the dye by using the Kubelka-Munk equation: ${ }^{8}$

$$
\frac{K}{S}=\frac{\left(1-R^{2}\right)}{2 R}=a q
$$

where $K$ is the absorbance coefficient of the fabric to be tested; $S$ is the scattering coefficient of the fabric to be tested; $R$ is the reflectance of the fabric at each wavelength; $a$ is a constant and $q$ is the adsorbed dye concentration on the fabric. The $K / S$ values from the fabric samples can be calculated for measurements made in a reflectance mode, and are directly correlated to the dye concentration on the dye substrate. The data shown for each sample were averages of three single measurements.

2.2.4 Colorfastness test. Colorfastness to laundering was measured at $49^{\circ} \mathrm{C}$ using AATCC Test Method 61 (AATCC 2013) by employing an Atlas LEF Launder-Ometer. A multi-fiber test fabric was attached to each sample to assess the staining. The test conditions were set according to the test no. 2A. Colorfastness to crocking was tested according to AATCC Test Method 8 (AATCC 2013). Both dry and wet crocking test were measured by employing a AATCC Automated Crockmeter. Colorfastness to light of the dyed samples was measured using test option 3 in AATCC Test Method 16.3 (AATCC 2014). The color change after 10 continuous light-on operating hours of each dyed sample was measured separately by employing an Atlas Ci 3000+ Weather-Ometer. For the whole colorfastness tests, the shade change and staining were evaluated according to AATCC Evaluation Procedure 1 (AATCC 2012) and AATCC Evaluation Procedure 2 (AATCC 2012) by employing grey scale ratings from 1 to 5 where 1 represents the biggest shade change while 5 implies no shade change. ${ }^{28}$

2.2.5 Flammability test. The flame-retardant test of the dyed samples was carried out by measuring the limiting oxygen index (LOI) value on a FAA flammability tester (ATSFAAR S.P.A,

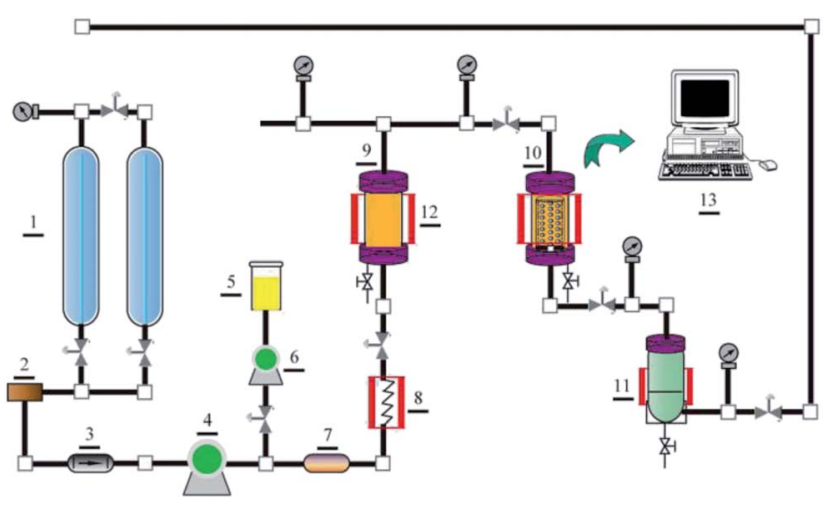

Fig. 1 Schematic diagram of the supercritical carbon dioxide dyeing apparatus. (1) $\mathrm{CO}_{2}$ cylinder, (2) purifier, (3) refrigerator, (4) high-pressure pump, (5) co-solvent tank, (6) co-solvent pump, (7) mixer, (8) heat exchanger, (9) dye vessel, (10) dyeing vessel, (11) separator, (12) heat compensating jacket, (13) computer. 
Italy) according to the standard ASTM D 2863-77, which was calculated by using eqn (2). ${ }^{24}$

$$
\mathrm{LOI}=\frac{V_{\text {oxygen }}}{V_{\text {nitrogen }}+V_{\text {oxygen }}} \times 100
$$

where $V_{\text {oxygen }}$ is the volume of oxygen; $V_{\text {nitrogen }}$ is the volume of nitrogen. In general, the higher a LOI value is, the better flammability will be observed.

\section{Results and discussion}

\subsection{Effect of dyeing temperature}

Temperature plays an important role in the dyeing of synthetic fibers with disperse dyes. In order to investigate the effect of dyeing temperature on color strength characterised as $K / S$ value of different disperse dyes on meta-aramid fabrics, the dyeing was carried out in supercritical carbon dioxide at a constant pressure of $30 \mathrm{MPa}$, a dyeing time of $70 \mathrm{~min}$, a dye concentration of $4.5 \%$ o.m.f. (on the mass of fabric), a carrier concentration of $3 \%$ o.m.f. (on the mass of carbon dioxide) for liquid and $3 \%$ o.m.f. (on the mass of fabric) for solid, as well as a carbon dioxide flow of $40 \mathrm{~g} \mathrm{~min}^{-1}$.

As shown in Fig. 2, when the meta-aramid fabric was dyed from $80{ }^{\circ} \mathrm{C}$ to $100{ }^{\circ} \mathrm{C}$ with Disperse Blue Black 79, Disperse Rubine H-2GL, and Disperse Yellow EC-3G in supercritical carbon dioxide, the $K / S$ values of the dyed samples stayed nearly constant due to the high degrees of crystallinity and orientation. After the dyeing temperature was increased to $140{ }^{\circ} \mathrm{C}$, the $\mathrm{K} / \mathrm{S}$ values on the dyed samples were improved gradually, and arrived the maximum. However, with the addition of different carriers, the $K / S$ values of the meta-aramid samples were increased obviously. The $K / S$ values of the dyed samples with ethyl alcohol were higher than the samples dyed with dimethyl terephthalate. Moreover, the highest change on $K / S$ values of the dyed $m e t a$-aramid was evidenced by adding CINDYE DNK in supercritical carbon dioxide.

In theory, thermal motion of disperse dye molecules and macromolecular chains of meta-aramid fiber were promoted under a higher temperature in supercritical carbon dioxide, thereby causing an increase of fiber free volume and benefiting the diffusion of dye molecules into the amorphous region of the fiber. ${ }^{5}$ In the dyeing process, the carrier mainly acts as a plasticizer, which can facilitate the mobility of segmental polymer chains, and result in the swelling of the fiber and a reduction in the glass transition temperature. ${ }^{8}$ Moreover, carrier can combine with meta-aramid fiber in the form of van der Waals force and hydrogen bonds, which could change the fiber-tofiber bonds into the fiber-to-carrier bonds, reducing the bonding force between the fiber and increasing the occurrence probability of the holes and the diffusion rate of disperse dye. Thus, the improvement in $K / S$ values of the dyed meta-aramid fabrics was significant with dimethyl terephthalate, ethyl alcohol, and CINDYE DNK in supercritical carbon dioxide in comparison with the samples without carriers. The biggest increase of $K / S$ values for meta-aramid samples with CINDYE DNK may be because CINDYE DNK is an aromatic amide compound. Based on like-dissolves-like theory, compared with
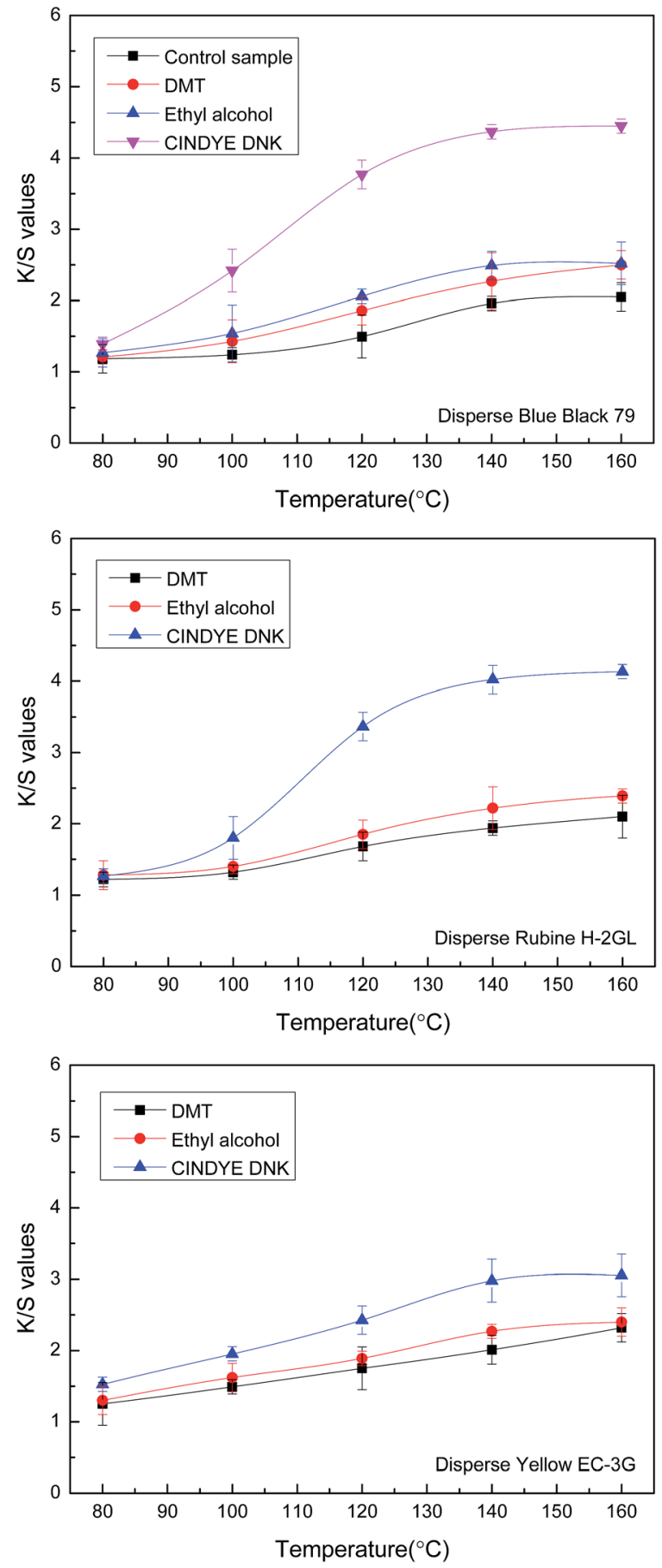

Fig. 2 Effect of dyeing temperature on $K / S$ values of meta-aramid fabrics.

other two carriers, it has a better expansion effect to metaaramid fiber in supercritical carbon dioxide. The moderate increase of $K / S$ values for meta-aramid samples with dimethyl terephthalate was observed, which indicated that solid carrier presents smaller swelling effect to meta-aramid than CINDYE DNK in supercritical carbon dioxide since the increase of dye solubility is lower. In addition, the highest $K / S$ values for metaaramid samples were obtained with Disperse Blue Black 79 since it presents a better substantivity than Disperse Rubine $\mathrm{H}^{-}$ 2GL, and Disperse Yellow EC-3G. 


\subsection{Effect of dyeing pressure}

An investigation of the effect of dyeing pressure on color strength of meta-aramid fabrics was carried out in supercritical carbon dioxide at a constant temperature of $140{ }^{\circ} \mathrm{C}$, a dyeing time of $70 \mathrm{~min}$, a dye concentration of $4.5 \%$ o.m.f. (on the mass of fabric), a carrier concentration of $3 \%$ o.m.f. (on the mass of carbon dioxide) for liquid and $3 \%$ o.m.f. (on the mass of fabric) for solid, as well as a carbon dioxide flow of $40 \mathrm{~g} \mathrm{~min}^{-1}$. As shown in Fig. 3, the $K / S$ values of the dyed meta-aramid fabrics were improved linearly with the dyeing pressures from $18 \mathrm{MPa}$ to $30 \mathrm{MPa}$. Compared with the $K / S$ curve of the meta-aramid fabrics dyed in supercritical carbon dioxide, it found that the $K / S$ values of the dyed meta-aramid samples with dimethyl terephthalate, ethyl alcohol, and CINDYE DNK in supercritical carbon dioxide were higher than that samples without carriers at the same pressure. Simultaneously, among all the carriers, the $K / S$ values of the dyed meta-aramid fabrics were increased most significantly with the addition of CINDYE DNK in supercritical carbon dioxide.

In principle, the density of the supercritical carbon dioxide can be controlled by adjusting pressure and temperature. With the rising of dyeing pressure, the density of the supercritical carbon dioxide fluid was increased, thus resulting in a better solubility of disperse dye. Furthermore, supercritical carbon dioxide can also be regarded as a molecular lubricant in the dyeing process. Intensive interactions between the carbon dioxide fluid and macro-chains of the polymers lead to the obvious swelling of the meta-aramid fiber under a higher system pressure. ${ }^{21,29}$ Furthermore, the swelling of the meta-aramid fiber is enhanced in the presence of carriers. In particular, the solubilities of carriers in supercritical carbon dioxide were also increased with the increase of fluid density, which further led to the swelling of meta-aramid. For the aforementioned reasons, more dissolved disperse dye molecules are able to adsorb on the surfaces of the fibers and then diffuse into the amorphous regions of meta-aramid. Therefore, all the promoting effects at a higher dyeing pressure resulted in an enhancing $K / S$ values on the meta-aramid fabrics in supercritical carbon dioxide from $18 \mathrm{MPa}$ and $30 \mathrm{MPa}$.

\subsection{Effect of dyeing time}

The effect of dyeing time on color strength of meta-aramid fabrics was investigated in supercritical carbon dioxide at a constant temperature of $140{ }^{\circ} \mathrm{C}$, a dyeing pressure of $30 \mathrm{MPa}$, a dye concentration of $4.5 \%$ o.m.f. (on the mass of fabric), a carrier concentration of $3 \%$ o.m.f. (on the mass of carbon dioxide) for liquid and 3\% o.m.f. (on the mass of fabric) for solid, as well as a carbon dioxide flow of $40 \mathrm{~g} \mathrm{~min}^{-1}$. It can be seen from Fig. 4 that the $K / S$ values of the dyed meta-aramid fabrics were improved notably from $10 \mathrm{~min}$ to $70 \mathrm{~min}$, which indicated that the rapid adsorption of the disperse dyes on the surface of meta-aramid occurred, and dye molecules diffused into the amorphous regions of the fiber within $70 \mathrm{~min}$ with the action of dimethyl terephthalate, ethyl alcohol, and CINDYE DNK. Then, a plateau appeared after $70 \mathrm{~min}$ on the dyeing curve, which displayed that near-saturation of dye adsorption was obtained over $70 \mathrm{~min}$ in supercritical carbon dioxide.
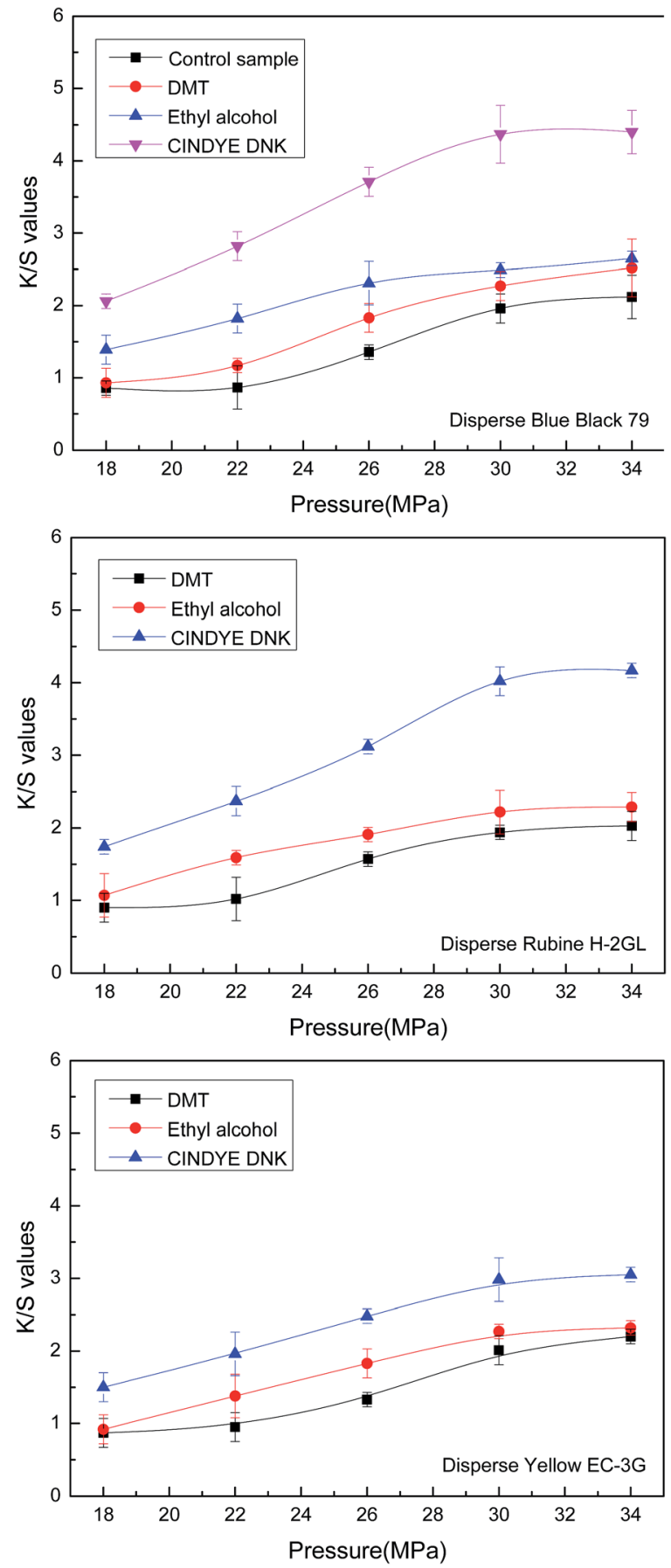

Fig. 3 Effect of dyeing pressure on K/S values of meta-aramid fabrics.

Furthermore, it also found from Fig. 4 that the $K / S$ values of the dyed meta-aramid samples with dimethyl terephthalate, ethyl alcohol and CINDYE DNK were higher in comparison with the $K / S$ value curve of the meta-aramid samples without carrier at the same dyeing time, which demonstrated that the swelling of meta-aramid with dimethyl terephthalate, ethyl alcohol and CINDYE DNK was improved gradually with the extended dyeing time in supercritical carbon dioxide. More disperse dye molecules were absorbed into fibers under the action of carriers, and the highest color strength for meta-aramid samples was achieved by employing Disperse Blue Black 79. Meanwhile, after 

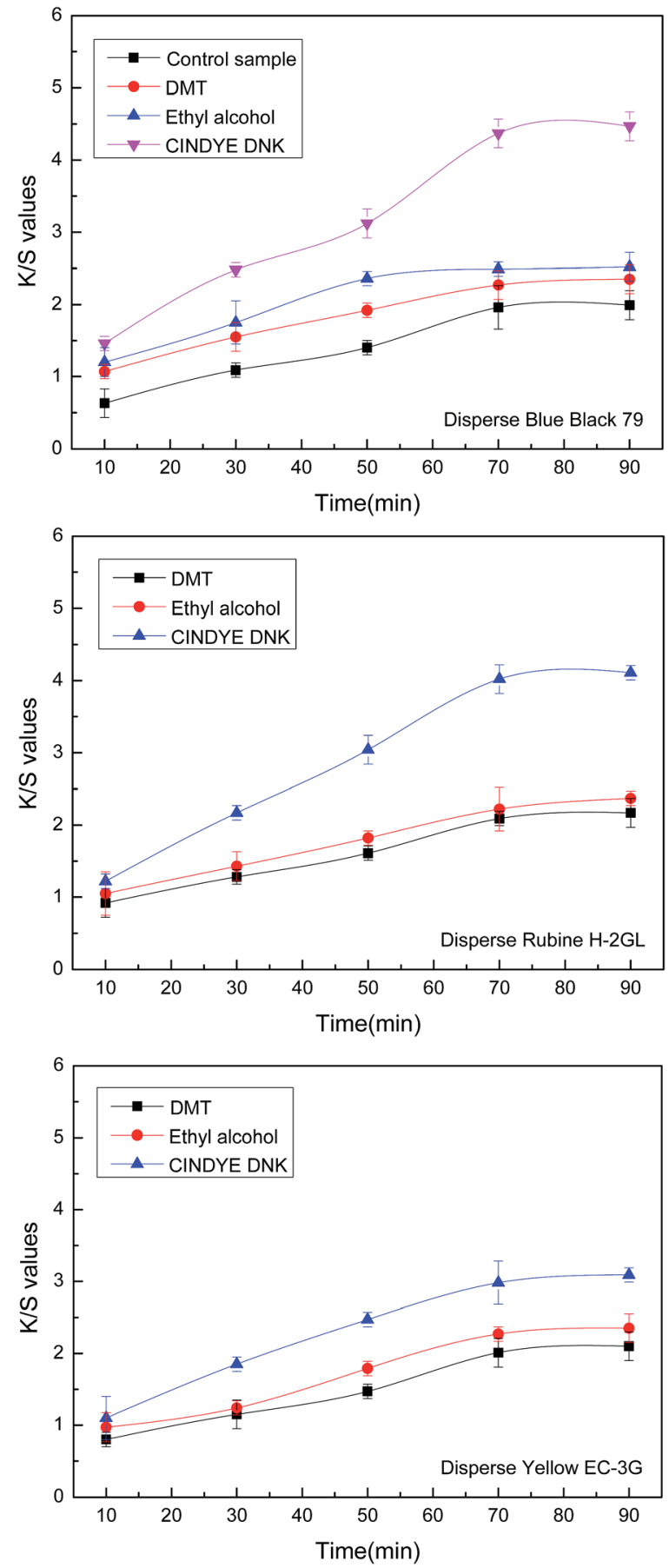

Fig. 4 Effect of dyeing time on $K / S$ values of meta-aramid fabrics.

supercritical carbon dioxide dyeing of $70 \mathrm{~min}$, the meta-aramid samples with CINDYE DNK presented the greatest improvement on $K / S$ values while the samples with dimethyl terephthalate showed the smallest improvement due to the lower swelling effect.

\subsection{Effect of dye concentration}

The effect of dye concentration on color strength of metaaramid fabrics was investigated in supercritical carbon dioxide at a constant temperature of $140{ }^{\circ} \mathrm{C}$, a dyeing pressure of $30 \mathrm{MPa}$, a dyeing time of $70 \mathrm{~min}$, a carrier concentration of $3 \%$ o.m.f. (on the mass of carbon dioxide) for liquid and $3 \%$ o.m.f. (on the mass of fabric) for solid, as well as a carbon dioxide flow of $40 \mathrm{~g} \mathrm{~min} \mathrm{~m}^{-1}$.

The results in Fig. 5 shown that the $K / S$ values of the dyed meta-aramid samples were improved enormously with the dye
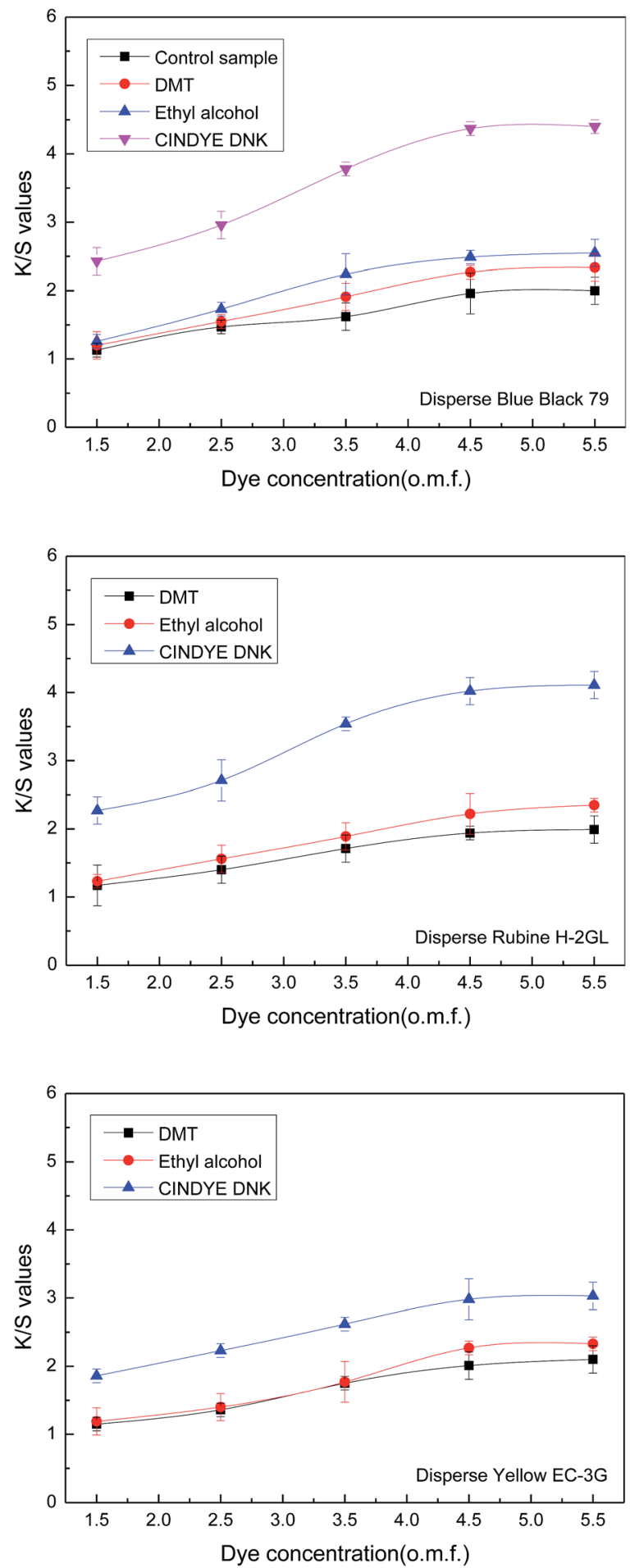

Fig. 5 Effect of dye concentration on $K / S$ values of meta-aramid fabrics. 
concentration increase from $1.5 \%$ o.m.f. to $4.5 \%$ o.m.f. under the presence of dimethyl terephthalate, ethyl alcohol, and CINDYE DNK, and a plateau was then reached at $4.5 \%$ o.m.f., which proved that better build up of Disperse Blue Black 79, Disperse Rubine H-2GL, and Disperse Yellow EC-3G was obtained in supercritical carbon dioxide dyeing process. Moreover, it also indicated that a certain quantity of meta-aramid fiber adsorbed a certain quantity of disperse dye molecules in
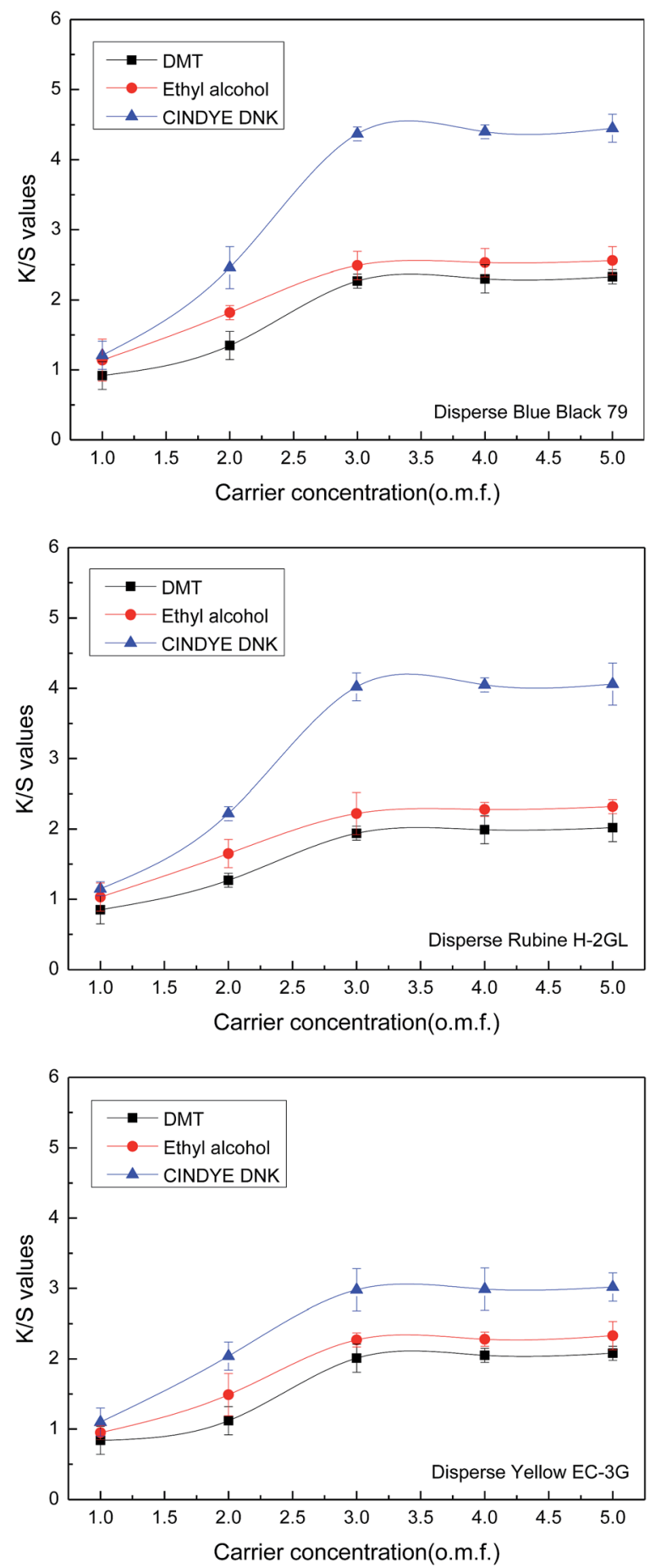

Fig. 6 Effect of carrier concentration on K/S values of meta-aramid fabrics. supercritical carbon dioxide because of the limited dye-sites in the fiber. During dyeing, excess dye molecules were dissolved in supercritical carbon dioxide when the dye quantity was greater than the quantity which could be absorbed into meta-aramid fiber. It means that dye utilization tends to be high at a low dye concentration. The dyes on meta-aramid fiber got saturation of adsorption with $4.5 \%$ o.m.f. in supercritical carbon dioxide using dimethyl terephthalate, ethyl alcohol, and CINDYE DNK as carriers.
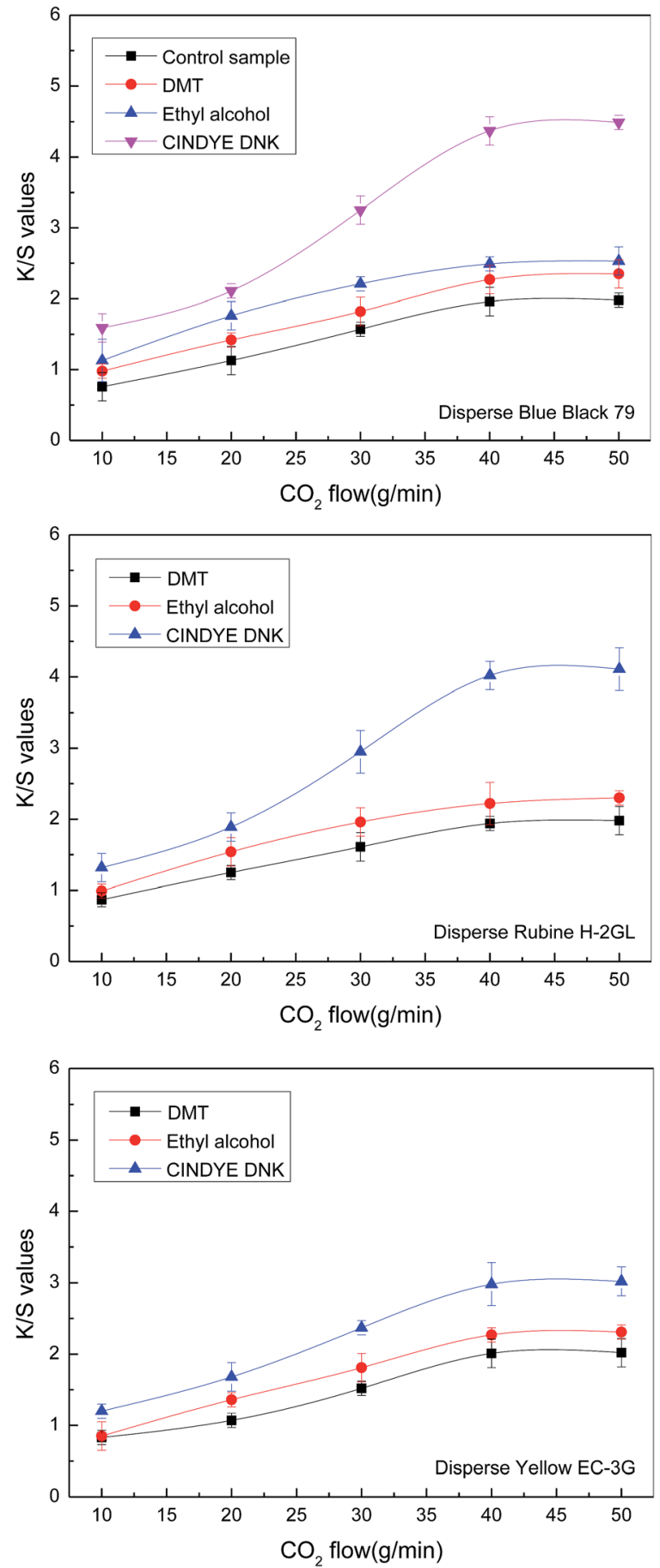

Fig. 7 Effect of carbon dioxide flow on K/S values of meta-aramid fabrics. 


\subsection{Effect of carrier concentration}

The effect of carrier concentration on color strength of metaaramid fabrics was investigated in supercritical carbon dioxide at a constant temperature of $140{ }^{\circ} \mathrm{C}$, a dyeing pressure of $30 \mathrm{MPa}$, a dyeing time of $70 \mathrm{~min}$, a dye concentration of $4.5 \%$ o.m.f. (on the mass of fabric), as well as a carbon dioxide flow of $40 \mathrm{~g} \min ^{-1}$.

Fig. 6 shows that the $K / S$ values of the dyed meta-aramid fabrics were enhanced with the increase of carrier concentration from $1 \%$ to $5 \%$ o.m.f. (on the mass of carbon dioxide). When a carrier concentration of $3 \%$ o.m.f. was reached, the $K / S$ values of the dyed samples were almost leveled off, which suggested that meta-aramid fabrics were almost saturated with dye molecules in this condition. At all selected concentrations, more carriers can dissolve in supercritical carbon dioxide. Moreover, carriers presents a higher affinity towards meta-aramid fiber, and result in the swelling of fibers. Hence, dye molecules in supercritical carbon dioxide are preferentially adsorbed onto the fiber surface, and formed a surface layer. As the dyeing process proceeds, dye molecules diffuse gradually from the surface layer into the amorphous regions of the meta-aramid fiber with the diffusing of the dimethyl terephthalate, ethyl alcohol, and CINDYE DNK.

\subsection{Effect of carbon dioxide flow}

The effect of carbon dioxide flow on color strength of metaaramid fabrics was investigated in supercritical carbon dioxide at a constant temperature of $140{ }^{\circ} \mathrm{C}$, a dyeing pressure of $30 \mathrm{MPa}$, a dyeing time of $70 \mathrm{~min}$, a dye concentration of $4.5 \%$ o.m.f. (on the mass of fabric), as well as a carrier concentration of $3 \%$ o.m.f. (on the mass of carbon dioxide) for liquid and $3 \%$ o.m.f. (on the mass of fabric) for solid. It can be seen from Fig. 7 that the $K / S$ values of the dyed meta-aramid fabrics were increased steadily as the carbon dioxide flow was increased from $10 \mathrm{~g} \mathrm{~min}^{-1}$ to $40 \mathrm{~g} \min ^{-1}$. This observation can be explained by the increase of the carrier concentration and the dye concentration with the rising of the carbon dioxide flow, which improved the swelling of fibers and the $K / S$ values.

At a constant temperature of $140{ }^{\circ} \mathrm{C}$ and a dyeing pressure of $30 \mathrm{MPa}$, the amount of carriers and dye molecules in supercritical carbon dioxide was increased in unit time. Thus, more dye molecules and carriers were able to penetrate into the amorphous phase of meta-aramid fiber, causing a better diffusion of the dye molecules into the fiber. In addition, after the carbon dioxide flow was further increased to $40 \mathrm{~g} \mathrm{~min}^{-1}$, an observation of the plateau on the $K / S$ curve indicated that the dwell time of the dyes and the carrier on the surface of metaaramid fiber was decreased due to the increase of carbon dioxide flow. Consequently, there was no longer increase for the $K / S$ values of the dyed meta-aramid fabrics in supercritical carbon dioxide.

\subsection{Functional mechanism of carriers}

The role of carriers in supercritical carbon dioxide is similar to polar cosolvent because of their polarity characteristic. As shown in Fig. 8, in the binary system of carrier and supercritical carbon dioxide, carrier molecules can form a large number of annular multimers to reduce their polarity, promoting the dissolution in supercritical carbon dioxide. Furthermore, the solubilities of carrier monomer and multimers were increased due to the weak hydrogen bonding interactions between carrier and supercritical carbon dioxide. ${ }^{30}$ More carrier molecules were dissolved in supercritical carbon dioxide, and absorbed on the surface of meta-aramid fiber with the circulation of carbon dioxide fluid.

As depicted in Fig. 9, CINDYE DNK is an aromatic amide compound, showing a similar structure with meta-aramid. Thus, CINDYE DNK displayed the better affinity towards macromolecular chain segment of meta-aramid in comparison with other two carriers. In supercritical carbon dioxide, carrier

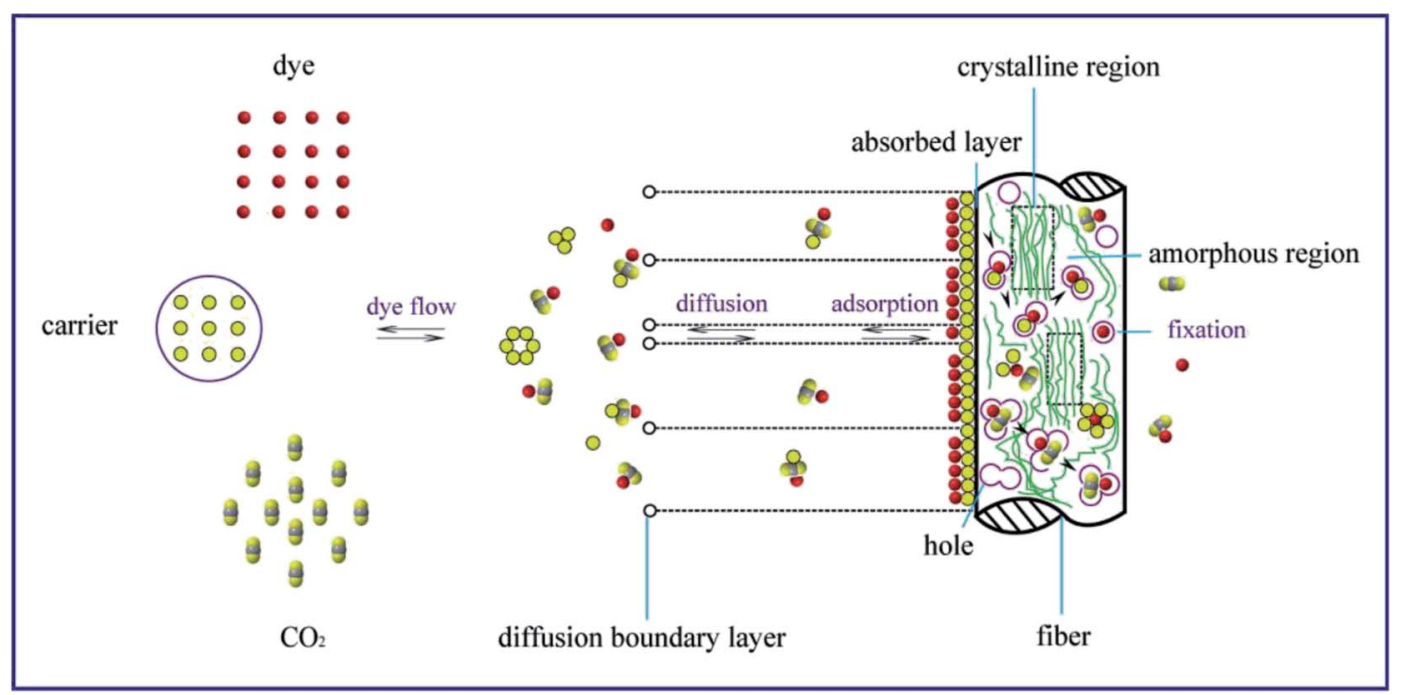

Fig. 8 Proposed functional mechanism of carriers in supercritical carbon dioxide. 


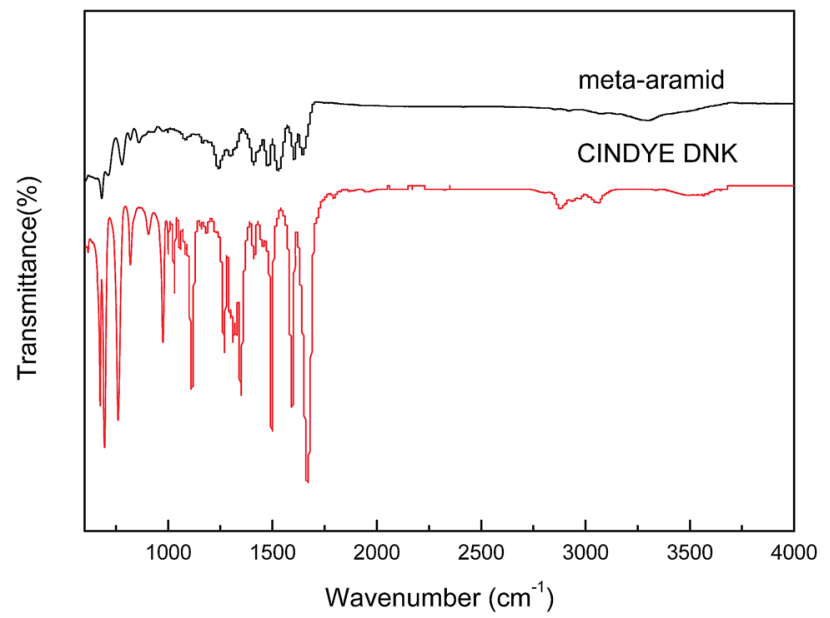

Fig. 9 FT-IR spectra of meta-aramid fibers and CINDYE DNK.

molecules diffused into the meta-aramid fiber, and combined with fiber macromolecules in the form of van der Waals force and hydrogen bonds, changing the fiber-to-fiber bonds into the fiber-to-carrier bonds. ${ }^{31}$ Therefore, the bonding force between the fiber macromolecules was decreased while the mobility of fiber macromolecule chain segments was increased, leading to the increase of the free volumes and occurrence probability of the holes in meta-aramid fiber. The diffusion rate and the dyeuptake of disperse dyes were increased accordingly. In the dyeing process, dye molecules gradually went through the diffusion boundary layer, diffused and absorbed on the surface of meta-aramid fiber under the attractive forces between molecules. Then, the dye molecules diffused along the holes in the fiber, and achieved the fixation in supercritical carbon dioxide.

\subsection{Colorfastness and flammability test}

The dyed meta-aramid samples with and without carrier were tested for colorfastness to laundering, crocking and light, and the results are summarized in Table 4 . Table 4 shows that the excellent dry and wet rub fastness, and staining fastness rated at
4-5 on an adjacent fabric of SDC Multifibre DW (wool, acrylic, polyester, polyamide-66, cotton and acetate) in wash testing experiments, were obtained for meta-aramid samples at a temperature of $140{ }^{\circ} \mathrm{C}$, a dyeing pressure of $30 \mathrm{MPa}$, a dyeing time of $70 \mathrm{~min}$, a dye concentration of $4.5 \%$ o.m.f. (on the mass of fabric), a carrier concentration of $3 \%$ o.m.f. (on the mass of carbon dioxide) for liquid and $3 \%$ o.m.f. (on the mass of fabric) for solid, as well as a carbon dioxide flow of $40 \mathrm{~g} \mathrm{~min}^{-1}$. Moreover, the dyed meta-aramid with carriers also showed the excellent light colorfastness, which was rated $4-5$. It is obvious that the rub colorfastness and light colorfastness of the dyed meta-aramid samples were improved in the presence of dimethyl terephthalate, ethyl alcohol, and CINDYE DNK during supercritical carbon dioxide dyeing process.

The effects of the carriers on the flammability of metaaramid samples are shown in Table 5. The data in Table 5 indicate that the LOI values for all the samples before and after supercritical carbon dioxide dyeing have not changed since the unfixed dyes and the carriers were already extracted with fresh carbon dioxide for $20 \mathrm{~min}$ after the dyeing process, which demonstrated that there was little influence of carriers on the flammability of meta-aramid after supercritical carbon dioxide dyeing.

Table 5 Limiting oxygen index (LOI) of the dyed meta-aramid fabrics in supercritical carbon dioxide fluid $\left(140{ }^{\circ} \mathrm{C}, 30 \mathrm{MPa}, 70 \mathrm{~min}, 40 \mathrm{~g}\right.$ $\mathrm{min}^{-1}$, a dye concentration of $4.5 \%$ o.m.f., and a carrier concentration of $3 \%$ o.m.f. for liquid and solid)

\begin{tabular}{lll}
\hline Dye & Samples & LOI (\%) \\
\hline \multirow{2}{*}{ Disperse Blue Black 79 } & Control sample & 28 \\
& DMT & 28 \\
& Ethyl alcohol & 28 \\
Disperse Rubine H-2GL & CINDYE DNK & 28 \\
& DMT & 28 \\
Disperse Yellow EC-3G & Ethyl alcohol & 28 \\
& CINDYE DNK & 28 \\
& DMT & 28 \\
& Ethyl alcohol & 28 \\
& CINDYE DNK & 28
\end{tabular}

Table 4 Colorfastness properties of dyed meta-aramid fabrics in supercritical carbon dioxide fluid

\begin{tabular}{|c|c|c|c|c|c|c|c|c|c|c|c|}
\hline \multirow[b]{3}{*}{ Dye } & \multirow[b]{3}{*}{ Samples } & \multicolumn{7}{|l|}{ Wash fastness } & \multicolumn{2}{|c|}{$\begin{array}{l}\text { Rub } \\
\text { fastness }\end{array}$} & \multirow[b]{3}{*}{ Light fastness } \\
\hline & & \multirow[b]{2}{*}{ Color change } & \multicolumn{6}{|c|}{ Staining } & \multirow[b]{2}{*}{ Dry } & \multirow[b]{2}{*}{ Wet } & \\
\hline & & & Wool & Acrylic & Polyester & Polyamide-66 & Cotton & Acetate & & & \\
\hline \multirow{4}{*}{ Disperse Blue Black 79} & Control sample & $4-5$ & $4-5$ & $4-5$ & $4-5$ & $4-5$ & $4-5$ & $4-5$ & $4-5$ & $4-5$ & $4-5$ \\
\hline & DMT & $4-5$ & $4-5$ & $4-5$ & $4-5$ & $4-5$ & $4-5$ & $4-5$ & 5 & 5 & 5 \\
\hline & Ethyl alcohol & $4-5$ & $4-5$ & $4-5$ & $4-5$ & $4-5$ & $4-5$ & $4-5$ & 5 & 5 & 5 \\
\hline & CINDYE DNK & $4-5$ & $4-5$ & $4-5$ & $4-5$ & $4-5$ & $4-5$ & $4-5$ & 5 & 5 & 5 \\
\hline \multirow[t]{3}{*}{ Disperse Rubine H-2GL } & $\mathrm{DMT}$ & $4-5$ & $4-5$ & $4-5$ & $4-5$ & $4-5$ & $4-5$ & $4-5$ & 5 & 5 & 5 \\
\hline & Ethyl alcohol & $4-5$ & $4-5$ & $4-5$ & $4-5$ & $4-5$ & $4-5$ & $4-5$ & 5 & 5 & 5 \\
\hline & CINDYE DNK & $4-5$ & $4-5$ & $4-5$ & $4-5$ & $4-5$ & $4-5$ & $4-5$ & 5 & 5 & 5 \\
\hline \multirow[t]{3}{*}{ Disperse Yellow EC-3G } & DMT & $4-5$ & $4-5$ & $4-5$ & $4-5$ & $4-5$ & $4-5$ & $4-5$ & 5 & 5 & 5 \\
\hline & Ethyl alcohol & $4-5$ & $4-5$ & $4-5$ & $4-5$ & $4-5$ & $4-5$ & $4-5$ & 5 & 5 & 5 \\
\hline & CINDYE DNK & $4-5$ & $4-5$ & $4-5$ & $4-5$ & $4-5$ & $4-5$ & $4-5$ & 5 & 5 & 5 \\
\hline
\end{tabular}




\section{Conclusions}

The present work investigated the dyeing properties of metaaramid fabrics with dimethyl terephthalate, ethyl alcohol and CINDYE DNK as carriers in supercritical carbon dioxide. The functional mechanism of carriers in supercritical carbon dioxide was proposed. The results indicated that dyeing temperature, dyeing pressure, dyeing time, dye concentration, carrier concentration and carbon dioxide flow would be beneficial in improving the dyeability of meta-aramid fabrics with Disperse Blue Black 79, Disperse Rubine H-2GL, and Disperse Yellow EC-3G. The carriers selected in supercritical carbon dioxide could intensify the movement of macromolecular chains of meta-aramid, reduce the glass transition temperature of fiber, and increase the diffusibility of dye molecules into the amorphous phase of the fiber, thus improving the dyeing properties of meta-aramid samples. The colorfastness data showed that good wash fastness (color change and stain), rubbing fastness (wet and dry) and light fastness were obtained for the meta-aramid samples dyed in supercritical carbon dioxide. In addition, there was little influence of carriers on the flammability of meta-aramid. Therefore, it is an effective and advantageous solution to dye meta-aramid with non-toxic carriers in supercritical carbon dioxide to improve the dyeability.

\section{Acknowledgements}

The authors would like to thank the financial support from Program of High-end Foreign Experts Working in the Educational and Cultural Sector (No. GDW20162100068), Scholarship from China Scholarship Council (No. 201406790023), and Group Project of Liaoning Provincial Department of Education (No. 2016J003).

\section{References}

1 A. Ferri, M. Banchero, L. Manna and S. Sicardi, J. Supercrit. Fluids, 2004, 30, 41-49.

2 A. Özcan, A. Clifford, K. Bartle and D. Lewis, Dyes Pigm., 1998, 36, 103-110.

3 H. Zheng, J. Zhang, J. Yan and L. Zheng, J. CO ${ }_{2}$ Util., 2016, 16, 272-281.

4 T. Hori and A. Kongdee, Dyes Pigm., 2014, 105, 163-166.

5 J. Long, Y. Ma and J. Zhao, J. Supercrit. Fluids, 2011, 57, 8086.

6 M. Banchero, Color. Technol., 2012, 129, 1-16.
7 X. Zhang, S. Heinonen and E. Levänen, RSC Adv., 2014, 4, 61137-61152.

8 L. Zheng, H. Zheng, B. Du, J. Wei, S. Gao and J. Zhang, J. Eng. Fibers Fabr., 2015, 10, 37-46.

9 D. Gao, D. Yang, H. Cui, T. Huang and J. Lin, ACS Sustainable Chem. Eng., 2015, 3, 668-674.

10 T. Abou Elmaaty, J. Ma, F. El-Taweel, E. Abd El-Aziz and S. Okubayashi, Ind. Eng. Chem. Res., 2014, 53, 15566-15570.

11 A. Özcan and A. Özcan, J. Supercrit. Fluids, 2005, 35, 133-139.

12 H. Wen and J. Dai, J. Appl. Polym. Sci., 2007, 105, 1903-1907.

13 S. Liao, J. Polym. Res., 2004, 11, 285-291.

14 S. Liao, Y. Ho and P. Chang, Color. Technol., 2000, 116, 403407.

15 T. Elmaaty, E. El-Aziz, J. Ma, F. El-Taweel and S. Okubayashi, Fibers, 2015, 3, 309-322.

16 Z. Liu, L. Zhang, Z. Liu, Z. Gao, W. Dong, H. Xiong, Y. Peng and S. Tang, Ind. Eng. Chem. Res., 2006, 45, 8932-8938.

17 A. Schmidt, E. Bach and E. Schollmeyer, Dyes Pigm., 2003, 56, 27-35.

18 M. Cid, M. Kraan, W. Veugelers, G. Woerlee and G. Witkamp, J. Supercrit. Fluids, 2004, 32, 147-152.

19 J. Fink, High Performance Polymer, Norwich: William Andrew Inc, 2008, pp. 423-448.

20 S. Zulfiqar and M. Sarwar, Scr. Mater., 2008, 59, 436-439.

21 H. Zheng, J. Zhang, B. Du, Q. Wei and L. Zheng, Fibers Polym., 2015, 16, 1134-1141.

22 H. Zheng, J. Zhang, B. Du, Q. Wei and L. Zheng, J. Appl. Polym. Sci., 2015, 132, 41756.

23 Y. Mao, Y. Guan, Q. Zheng, Q. Liu, X. Feng and X. Wang, Color. Technol., 2012, 129, 39-48.

24 Y. Mao, Y. Guan and P. Zhu, Korean J. Chem. Eng., 2015, 32, 2133-2141.

25 L. Lei, Y. Mao, X. Xu, J. Zheng, Q. Zheng, Y. Guan and X. Fan, Color. Technol., 2014, 130, 349-356.

26 A. Nechwatal and V. Rossbach, Text. Res. J., 1999, 69, 635640.

27 Chemicalland21, http:/www.chemicalland21.com/, accessed September 2016.

28 S. Fu, D. Hinks, P. Hauser and M. Ankeny, Cellulose, 2013, 20, 3101-3110.

29 H. Kong, C. Teng, X. Liu, J. Zhou, H. Zhong, Y. Zhang, K. Han and M. Yu, RSC Adv., 2014, 4, 20599-20604.

$30 \mathrm{~W}$. Xu, Computer simulations for supercritical carbon dioxide systems, PhD thesis, Tsinghua University, China, 2010.

31 P. Liang and L. Wang, Dyest. Color., 2010, 47, 24-27. 